\title{
FORMULATION OF VALUE ADDED CHICKEN MEATBALL WITH DIFFERENT LEVEL OF WHEAT FLOUR
}

\author{
M.A. Islam ${ }^{1}$, M.A. Haque ${ }^{2 *}$, M.J. Ferdwsi ${ }^{3}$, M.Y. Ali ${ }^{4}$ and M.A. Hashem ${ }^{1}$ \\ ${ }^{1}$ Department of Animal Science, Faculty of Animal Husbandry, Bangladesh Agricultural University, \\ Mymensingh 2202, Bangladesh \\ ${ }^{2}$ Department of Biotechnology, Yeungnam University, Gyeongsan 38541, Republic of Korea \\ ${ }^{3}$ Faculty of Animal Husbandry, Bangladesh Agricultural University, Mymensingh 2202, Bangladesh \\ ${ }^{4}$ Goat and Sheep Production Research Division, Bangladesh Livestock Research Institute, Savar, \\ Bangladesh
}

\begin{abstract}
The present study was undertaken to evaluate the effect of different levels of wheat flour on the quality characteristics of chicken meatball. Wheat flour which acts as a binding agent of meatball except for control group $T_{1}$. The meatballs were formulated having $0 \%, 5 \%, 10 \%$ and $15 \%$ wheat flour. The sensory (colour, flavour, texture, juiciness, tenderness, overall acceptability), physicochemical (proximate analysis, $\mathrm{p}^{\mathrm{H}}$, cooking loss), biochemical (TBARs, POV, FFA) were analyzed. Treatments were analyzed in a $4 \times 3$ factorial experiment in CRD replicated three times per cell. Wheat flour inclusion in meatballs increased cooking yield by reducing weight loss from 27.06 to $26.49 \%$. Among four treatments most preferable colour, odour, tenderness, juiciness was observed significantly $(p<0.05)$ at $15 \%$ wheat flour group and the less preferable colour was observed from the control group. The preferablecolourwas observed at 0 days and less preferable colour at 30 day. Meatballs made with the addition of $15 \%$ wheat flour had the highest tenderness, overall acceptability, raw $\mathrm{p}^{\mathrm{H}}$, cooked $\mathrm{p}^{\mathrm{H}}$ and lower DM, ash, PV and TBA \& showed significant value $(p<0.05)$ The cooked $p^{H}$ was decreased with the increased storage period. Meatballs with wheat flour inclusions at $15 \%$ were most acceptable. It is recommended that further studies of the wheat flour inclusion in meatballs production be carried out to ensure the availability of cheaper, nutritious and acceptable convenience food in the Bangladeshi market.
\end{abstract}

Keywords: Meatballs, chicken, wheat flour, fibre and prebiotics.

\footnotetext{
"Corresponding author email: ah36482@bau.edu.bd
} 


\section{INTRODUCTION}

Chicken meat has gained much popularity among consumers and the consumption rate of chicken meat and chicken meat products is increasing day by day throughout the world. Among the different meat products, the meatball is one of the tasty and popular food's item. Meatballs can be made with beef, lamb, veal, pork, turkey, chicken and even offal. Meatball is a small ball of chopped or ground meat often mixed with bread crumbs and spices. For economic benefits, the substitution of beef in meatball with the meat of lower price such as chicken takes place frequently. The addition of fat replacers may lead to decreased energy value and cholesterol contents. Fat contributes key sensory benefits to foods and is perceived through mouthfeel, taste, and aroma/odour (Sampaio et al., 2004). Therefore, the reduction of fat content may have a large effect on the quality attributes of meat products such as colour, flavour, texture and binding properties. For this reason, ideal sources of fat replacers are needed to improve the functional value of meat products. Non-meat ingredients play a significant role in the modification of functional properties such as emulsification, and water and fat binding capacity, which may impact the textural properties. In the past, starch was added as a source of carbohydrates and to thicken the texture of meatballs by Huda et al. (2009). Today, starch is extensively used as a stabilizer, texturizer, water or fat binder and emulsifier. Apart from these functions, starch can also increase the gel strength and freeze-thaw stability of meatballs if added to appropriate levels by Serdaroglu et al. (2005). The aims of this study were to investigate the suitable level of wheat flour adding and its effects on the proximate composition, physicochemical properties and sensory qualities of chicken meatballs.

\section{MATERIALS AND METHODS}

\section{Sample preparation}

Boneless broiler meat of $2.5 \mathrm{~kg}$ from the freshly slaughtered chicken was collected from Bangladesh Agricultural University Poultry Farm, Mymensingh. The meat was ground properly and the spices, garam masala, salt, Ice flakes, refined vegetable oil, refined wheat flour, the sauce was mixed with the ground meat properly as per experimental design. There were four treatment groups are treated as a $\mathrm{T}_{1}$-control group (no flour), $\mathrm{T}_{2}-5 \%$ flour, $\mathrm{T}_{3}-10 \%$ flour, and $\mathrm{T}_{4}-15 \%$ flour. Then meatball of proper shape was prepared separately. It was then boiled in hot water for 2-3 minutes. Then the water was removed from the meatball properly and was fried in hot oil until the reddish brown colour was obtained.

\section{Sensory evaluation}

Sensory evaluation was carried out in individual booths under controlled conditions of light, temperature and humidity. Prior to sample evaluation, all panellists participated in orientation sessions to familiarize with the scale attributes (colour, smell, juiciness, tenderness, overall acceptability) of meatball using an intensity scale. Sensory qualities of the samples were evaluated after thawing of before cook 
and after cook using a 5-point scoring method. Sensory scores were 5 for excellent, 4 for very good, 3 for good, 2 for fair and 1 for poor. Sensory evaluation was accomplished at 0-day and repeated at 15, 30 and 60 day; up to the end of refrigerated storage at $-20 \pm 1^{\circ} \mathrm{C}$.

\section{Proximate composition}

A proximate composition such as dry matter (DM), ether extract (EE), crude protein (CP) and ash was carried out according to the methods (AOAC, 2005).

\section{Biochemical analysis}

Three types of biochemical analysis were practised in this research. The free fatty acid value was determined according to Rukunudin et al. (1998). Peroxide value (POV) was determined according to Sallam et al. (2004). Lipid oxidation was assessed in triplicate using the 2-thiobarbituric acid (TBA) method described by Schmedes and Holmer (1989).

\section{Physicochemical properties measurement}

$\mathrm{P}^{\mathrm{H}}$ value of raw and cooked meatball was measured using $\mathrm{p}^{\mathrm{H}}$ meter from raw meatball homogenate. The homogenate was prepared by blending $5 \mathrm{~g}$ of meat with 10 $\mathrm{ml}$ distilled water. Cooking loss of chicken meatball also determined.

\section{Statistical model and analysis}

The proposed model for the planned experiment was a factorial experiment with two factors A(Treatments)and $B$ (Days of Intervals)is:

$y i j k=\mu+A i+B j+(A B) i j+\varepsilon i j k i=1, \ldots, a ; j=1, \ldots, b ; k=1, \ldots, n$

Where,

$y i j k=$ observation $k$ in level $i$ of factor $A$ and level $j$ of factor $B$

$\mu=$ the overall mean

$A i=$ the effect of level $i$ of factor $A$

$B j=$ the effect of level $j$ of factor $B$

Data were statistically analyzed using SAS Statistical Discovery software, NC, USA. DMRT test was used to determine the significance of differences among treatments means.

\section{RESULTS AND DISCUSSION}

\section{Sensory evaluation}

Mean scores for different sensory attributes obtained from the sensory evaluation are shown in table 1.Thepreferablecolour was observed at 0-day. The decreased colour test scores during storage resulted from the denaturation of proteins, particularly the myofibrillar protein that affects gel formation. Among four treatments significantly $(p<0.05)$ higher colour score was observed in $15 \%$ wheat flour group than other 
treatments which are collaborating with the finding of Naveena et al. (2008). Preferable good flavour was observed in $15 \%$ wheat flour group and the quality was deteriorated with increased storage period. Among these four treatments most preferable tenderness was observed at $15 \%$ wheat flour group at 0-day which is similar with the findings of Ali and Zahran (2010) also reported that supplementation improved chicken meat tenderness during storage. Ngapo et al. (2004) stated that juiciness as an indicator of meatballs freshness or even eating quality. The range of overall observed juiciness score at different treatments was 4.11 to 4.56 . The preferableacceptability was observed at 0 day. The data show that the lowest test score was reduced to 3.83 in all treatments after 30 days of storage.

Table 1. Effect of wheat flour on sensory parameters in chicken meatballs

\begin{tabular}{|c|c|c|c|c|c|c|c|c|c|}
\hline \multirow[t]{2}{*}{ Parameters } & \multirow[t]{2}{*}{$\mathrm{DI}$} & \multicolumn{4}{|c|}{ Treatments } & \multirow[t]{2}{*}{ Mean } & \multicolumn{3}{|c|}{$\begin{array}{c}\text { Level of } \\
\text { significance }\end{array}$} \\
\hline & & $\mathrm{T}_{1}$ & $\mathrm{~T}_{2}$ & $\mathrm{~T}_{3}$ & $\mathrm{~T}_{4}$ & & Treat. & DI & $\mathrm{T} \times \mathrm{DI}$ \\
\hline \multirow{3}{*}{ Color } & 0 & $4.33 \pm 0.33$ & $4.66 \pm 0.33$ & $4.67 \pm 0.33$ & $4.67 \pm 0.33$ & $4.58^{\mathrm{a}} \pm 0.33$ & \multirow{3}{*}{0.595} & \multirow{3}{*}{0.013} & \multirow{3}{*}{0.695} \\
\hline & 30 & $4.33 \pm 0.33$ & $3.66 \pm 0.33$ & $4.00 \pm 0.58$ & $4.33 \pm 0.33$ & $3.83^{\mathrm{b}} \pm 0.39$ & & & \\
\hline & 60 & $4.67 \pm 0.33$ & $4.33 \pm 0.33$ & $4.67 \pm 0.33$ & $4.33 \pm 0.33$ & $4.50^{\mathrm{a}} \pm 0.33$ & & & \\
\hline Mean & & $4.11^{\mathrm{a}} \pm 0.33$ & $4.22^{\mathrm{a}} \pm 0.33$ & $4.44^{\mathrm{a}} \pm 0.41$ & $4.44^{\mathrm{a}} \pm 0.33$ & & & & \\
\hline \multirow{3}{*}{ Flavor } & 0 & $4.33 \pm 0.33$ & $4.67 \pm 0.33$ & $4.67 \pm 0.33$ & $5.00 \pm 0.00$ & $4.67^{\mathrm{a}} \pm 0.25$ & & & \\
\hline & 30 & $3.33 \pm 0.33$ & $3.67 \pm 0.33$ & $4.00 \pm 0.58$ & $4.33 \pm 0.33$ & $3.83^{\mathrm{b}} \pm 0.39$ & \multirow{2}{*}{0.165} & \multirow{2}{*}{0.005} & \multirow{2}{*}{0.926} \\
\hline & 60 & $4.33 \pm 0.33$ & $4.67 \pm 0.33$ & $4.33 \pm 0.33$ & $4.67 \pm 0.33$ & $4.50^{\mathrm{a}} \pm 0.33$ & & & \\
\hline \multirow[t]{2}{*}{ Mean } & & $4.00^{\mathrm{b}} \pm 0.33$ & $4.33^{\mathrm{ab}} \pm 0.33$ & $4.33^{\mathrm{ab}} \pm 0.41$ & $4.67^{\mathrm{a}} \pm 0.22$ & & & & \\
\hline & 0 & $4.67 \pm 0.33$ & $4.67 \pm 0.33$ & $5.00 \pm 0.00$ & $4.67 \pm 0.33$ & $4.80^{\mathrm{a}} \pm 0.25$ & & & \\
\hline \multirow[t]{2}{*}{ Tenderness } & 30 & $3.33 \pm 0.33$ & $4.00 \pm 0.00$ & $4.33 \pm 0.33$ & $4.67 \pm 0.33$ & $4.08^{\mathrm{b}} \pm 0.25$ & \multirow{2}{*}{0.145} & \multirow{2}{*}{0.001} & \multirow{2}{*}{0.071} \\
\hline & 60 & $4.33 \pm 0.33$ & $3.33 \pm 0.33$ & $4.33 \pm 0.33$ & $3.67 \pm 0.33$ & $3.92^{\mathrm{b}} \pm 0.33$ & & & \\
\hline Mean & & $4.11^{\mathrm{ab}} \pm 0.33$ & $4.00^{\mathrm{b}} \pm 0.22$ & $4.56^{\mathrm{a}} \pm 0.22$ & $4.33^{\mathrm{ab}} \pm 0.33$ & & & & \\
\hline \multirow{3}{*}{$\begin{array}{l}\text { Overall } \\
\text { Acceptability }\end{array}$} & 0 & $4.33 \pm 0.33$ & $4.67 \pm 0.33$ & $4.67 \pm 0.33$ & $4.67 \pm 0.33$ & $4.58^{\mathrm{a}} \pm 0.33$ & \multirow{3}{*}{0.431} & \multirow{3}{*}{0.024} & \\
\hline & 30 & $3.33 \pm 0.33$ & $3.67 \pm 0.33$ & $4.00 \pm 0.58$ & $4.33 \pm 0.33$ & $3.83^{\mathrm{ab}} \pm 0.39$ & & & \multirow{2}{*}{0.433} \\
\hline & 60 & $4.67 \pm 0.33$ & $3.67 \pm 0.33$ & $4.33 \pm 0.33$ & $4.33 \pm 0.33$ & $4.25^{\mathrm{ab}} \pm 0.33$ & & & \\
\hline \multirow[t]{2}{*}{ Mean } & & $4.11^{\mathrm{a}} \pm 0.33$ & $4.00^{\mathrm{a}} \pm 0.33$ & $4.33^{\mathrm{a}} \pm 0.41$ & $4.44^{\mathrm{a}} \pm 0.33$ & & \multirow{4}{*}{0.591} & \multirow{4}{*}{0.050} & \multirow{4}{*}{0.854} \\
\hline & 0 & $4.67 \pm 0.30$ & $4.67 \pm 0.33$ & $4.33 \pm 0.33$ & $5.00 \pm 0.00$ & $4.67^{\mathrm{a}} \pm 024$ & & & \\
\hline \multirow[t]{2}{*}{ Juiciness } & 30 & $3.67 \pm 067$ & $3.67 \pm 0.33$ & $4.00 \pm 0.58$ & $4.33 \pm 0.33$ & $3.92^{\mathrm{b}} \pm 0.48$ & & & \\
\hline & 60 & $4.33 \pm 0.33$ & $4.67 \pm 0.33$ & $4.00 \pm 0.58$ & $4.33 \pm 0.33$ & $4.33^{\mathrm{ab}} \pm 0.39$ & & & \\
\hline Mean & & $4.22^{\mathrm{a}} \pm 043$ & $4.33^{\mathrm{a}} \pm 0.33$ & $4.11^{\mathrm{a}} \pm 0.50$ & $4.56^{\mathrm{a}} \pm 022$ & & & & \\
\hline
\end{tabular}

Column means having different superscript varies significantly at values $p<0.05$. Again, mean values having the same superscript in each row did not differ significantly at $\mathrm{p}>0.05 . \mathrm{T}_{1}=$ Control group, $\mathrm{T}_{2}=$ $05 \%$ wheat flour group, $\mathrm{T}_{3}=10 \%$ wheat flour group, $\mathrm{T}_{4}=15 \%$ wheat flour group, DI=Day Intervals, Treat $=$ Treatment, $\mathrm{T} \times \mathrm{DI}=$ Interaction of Treatment and Day Intervals. 


\section{Proximate analysis}

The dry matter content was increased with the increased storage period because moisture loss was decreased with the storage period (Table 2). Similar results were reported for Indonesian traditional meatballs with a dry matter content ranged from 56.17 to $60.32 \%$ mentioned by Purnomo and Rahardiyan (2008). The data showed that the highest amount of CP content was 20.70 in all treatments at 0 days of storage and the lowest amount of CP content was found after 30 days of storage. The protein result was lower compared to the protein content of Indonesian beef meatballs which ranged from 13.38 to $14.44 \%$ reported by Purnomo and Rahardiyan et.al. (2008). The highest amount of EE content was increased to $8.52 \%$ in all treatments after 0 day of storage. The Malaysian Food Regulation of 1985 stated that manufactured meat should not contain more than $30 \%$ fat. Malaysian beef meatballs can be classified as low-fat meatballs since the fat content ranges from 1.69 to $11.09 \%$. The ash content was significantly changed with the increased storage period.The data show that the highest amount of Ash content was increased to $1.35 \%$ in all treatments after 60 days of storage.

Table 2. Effects of wheat flour on proximate components in chicken meatballs

\begin{tabular}{|c|c|c|c|c|c|c|c|c|c|}
\hline \multirow{2}{*}{ Parameters } & \multirow{2}{*}{ DI } & \multicolumn{4}{|c|}{ Treatments } & \multirow{2}{*}{ Mean } & \multicolumn{3}{|c|}{ Level of significance } \\
\hline & & $\mathrm{T}_{1}$ & $\mathrm{~T}_{2}$ & $\mathrm{~T}_{3}$ & $\mathrm{~T}_{4}$ & & Treat. & DI & $\mathrm{T} \times \mathrm{DI}$ \\
\hline \multirow{3}{*}{$\operatorname{DM}(\%)$} & 0 & $53.73 \pm 0.10$ & $47.33 \pm 0.14$ & $45.74 \pm 0.08$ & $54.12 \pm 0.09$ & $50.23^{\mathrm{a}} \pm 0.10$ & & & \\
\hline & 30 & $51.20 \pm 0.62$ & $48.14 \pm 0.19$ & $53.78 \pm 0.01$ & $47.27 \pm 0.43$ & $50.10^{\mathrm{a}} \pm 0.31$ & \multirow{2}{*}{\multicolumn{3}{|c|}{$<0.001<0.001<0.001$}} \\
\hline & 60 & $51.65 \pm 0.52$ & $49.64 \pm 0.58$ & $46.59 \pm 0.37$ & $47.31 \pm 0.30$ & $48.80^{\mathrm{b}} \pm 0.44$ & & & \\
\hline \multirow[t]{2}{*}{ Mean } & & $52.19^{\mathrm{a}} \pm 0.42$ & $48.37^{c} \pm 0.30$ & $48.7^{\mathrm{c}} \pm 0.13$ & $49.56^{\mathrm{b}} \pm 0.27$ & & & & \\
\hline & 0 & $21.42 \pm 0.29$ & $20.89 \pm 0.17$ & $20.21 \pm 0.13$ & $20.21 \pm 0.01$ & $20.70^{\mathrm{a}} \pm 0.17$ & & & \\
\hline \multirow[t]{2}{*}{$\mathrm{CP}(\%)$} & 30 & $21.10 \pm 0.45$ & $20.29 \pm 0.13$ & $19.75 \pm 0.19$ & $20.12 \pm 0.04$ & $20.32^{\mathrm{b}} \pm 0.20$ & \multirow{2}{*}{$<0.001$} & 00750 & 07541 \\
\hline & 60 & $21.37 \pm 0.07$ & $20.65 \pm 0.11$ & $19.74 \pm 0.32$ & $19.71 \pm 0.48$ & $20.36^{\mathrm{ab}} \pm 0.25$ & & 0.07 & 0.104 \\
\hline \multirow[t]{2}{*}{ Mean } & & $21.20^{\mathrm{a}} \pm 0.27$ & $20.61^{\mathrm{b}} \pm 0.14$ & $19.89^{c} \pm 0.21$ & $20.04^{\mathrm{c}} \pm 0.21$ & & & & \\
\hline & 0 & $7.88 \pm 0.27$ & $8.57 \pm 0.33$ & $8.93 \pm 0.14$ & $8.71 \pm 0.10$ & $8.52^{\mathrm{a}} \pm 0.21$ & & & \\
\hline \multirow[t]{2}{*}{$\mathrm{EE}(\%)$} & 30 & $8.26 \pm 0.02$ & $7.77 \pm 0.07$ & $8.52 \pm 0.06$ & $7.55 \pm 0.07$ & $8.03^{\mathrm{b}} \pm 0.06$ & \multirow{2}{*}{0.0078} & \multirow{2}{*}{0.0180} & \multirow{2}{*}{0.0270} \\
\hline & 60 & $8.00 \pm 0.39$ & $8.60 \pm 0.37$ & $8.44 \pm 0.30$ & $7.66 \pm 0.19$ & $8.18^{\mathrm{b}} \pm 0.31$ & & & \\
\hline \multirow[t]{2}{*}{ Mean } & & $8.05^{\mathrm{b}} \pm 0.23$ & $8.31^{\mathrm{ab}} \pm 0.26$ & $8.63^{\mathrm{a}} \pm 0.17$ & $7.97^{\mathrm{b}} \pm 0.12$ & & & & \\
\hline & 0 & $1.16 \pm 0.02$ & $1.44 \pm 0.05$ & $1.63 \pm 0.04$ & $1.04 \pm 0.04$ & $1.32^{\mathrm{ab}} \pm 0.04$ & & & \\
\hline \multirow[t]{2}{*}{$\operatorname{ASH}(\%)$} & 30 & $1.24 \pm 0.05$ & $1.11 \pm 0.05$ & $1.24 \pm 0.09$ & $1.35 \pm 0.05$ & $1.23^{\mathrm{b}} \pm 0.24$ & \multirow{2}{*}{0.0590} & \multirow{2}{*}{0.0207} & \multirow{2}{*}{$<0.001$} \\
\hline & 60 & $1.39 \pm 0.02$ & $1.36 \pm 0.03$ & $1.27 \pm 0.11$ & $1.39 \pm 0.07$ & $1.35^{\mathrm{a}} \pm 0.23$ & & & \\
\hline Mean & & $1.26^{\mathrm{b}} \pm 0.03$ & $1.31^{\mathrm{ab}} \pm 0.04$ & $1.38^{\mathrm{a}} \pm 0.08$ & $1.26^{\mathrm{b}} \pm 0.05$ & & & & \\
\hline
\end{tabular}

Column means having different superscriptvaries significantly at values $\mathrm{p}<0.05$. Again, mean values having the same superscript in each row did not differ significantly at $\mathrm{p}>0.05$. $\mathrm{T}_{1}=$ Control group, $\mathrm{T}_{2}=$ $05 \%$ wheat flour group, $\mathrm{T}_{3}=10 \%$ wheat flour group, $\mathrm{T}_{4}=15 \%$ wheat flour group, DI=Day Intervals, Treat $=$ Treatment, $\mathrm{T} \times \mathrm{DI}=$ Interaction of Treatment and Day Intervals. 


\section{Physicochemical properties}

The raw and cooked meat mean $\mathrm{p}^{\mathrm{H}}$ was decreased with the increased storage period. Table 3 showed a slight decrease in the raw meat $\mathrm{p}^{\mathrm{H}}$ values and an increase in the acidity values for all samples along with storage time during the 60 days of storage as a result of the increase of free fatty acids due to rancidity. The preferable cooked meat $\mathrm{p}^{\mathrm{H}}$ content was observed from 0 -day and less preferable cooked $\mathrm{p}^{\mathrm{H}}$ was observed from 60 days observation. These results are similar to those of Sallam et al. (2004) who reported that storage time had a significant $(\mathrm{p}<0.05)$ effect on $\mathrm{p}^{\mathrm{H}}$ values, which tended to increase with storage time. The range of overall observed cooking loss at different treatments was 27.42 to $26.45 \%$. The cooking loss was decreased with the increased storage period. Major components of cooking losses are thawing, dripping and evaporation. The cooking loss in meat cuts is important for maintaining an attractive retail display of meat. The values of cooking yield were similar to the results in high-fat Kung-wan meatballs reported by Huang et al. (2005).

Table 3. Effect of wheat flour on physicochemicalparameters in chicken meatballs

\begin{tabular}{|c|c|c|c|c|c|c|c|c|c|}
\hline \multirow{2}{*}{ Parameters } & \multirow{2}{*}{ DI } & \multicolumn{4}{|c|}{ Treatments } & \multirow{2}{*}{ Mean } & \multicolumn{3}{|c|}{ Level of significance } \\
\hline & & $\mathrm{T}_{1}$ & $\mathrm{~T}_{2}$ & $\mathrm{~T}_{3}$ & $\mathrm{~T}_{4}$ & & Treat. & DI & $\mathrm{T} \times \mathrm{DI}$ \\
\hline \multirow{3}{*}{ Raw $\mathrm{P}^{\mathrm{H}}$} & 0 & $5.95 \pm 0.04$ & $5.94 \pm 0.04$ & $5.89 \pm 0.07$ & $5.84 \pm 0.10$ & $5.91^{\mathrm{a}} \pm 0.06$ & & & \\
\hline & 30 & $5.70 \pm 0.14$ & $5.89 \pm 0.17$ & $5.54 \pm 0.09$ & $5.53 \pm 0.06$ & $5.67^{\mathrm{b}} \pm 0.12$ & \multirow{2}{*}{\multicolumn{3}{|c|}{0.16900 .00280 .3431}} \\
\hline & 60 & $5.84 \pm 0.17$ & $5.41 \pm 0.18$ & $5.55 \pm 0.23$ & $5.44 \pm 0.07$ & $5.56^{\mathrm{b}} \pm 0.16$ & & & \\
\hline \multirow[t]{2}{*}{ Mean } & & $5.83^{\mathrm{a}} \pm 0.12$ & $5.74^{\mathrm{a}} \pm 0.13$ & $5.66^{\mathrm{a}} \pm 0.13$ & $5.60^{\mathrm{a}} \pm 0.08$ & & & & \\
\hline & 0 & $6.05 \pm 0.01$ & $6.06 \pm 0.03$ & $6.04 \pm 0.04$ & $6.07 \pm 0.06$ & $6.06^{\mathrm{a}} \pm 0.04$ & & & \\
\hline \multirow[t]{2}{*}{ Cooked $\mathrm{P}^{\mathrm{H}}$} & 30 & $6.02 \pm 0.01$ & $6.11 \pm 0.06$ & $6.02 \pm 0.01$ & $6.04 \pm 0.02$ & $6.05^{\mathrm{a}} \pm 0.03$ & \multirow{2}{*}{\multicolumn{3}{|c|}{0.81860 .89180 .4766}} \\
\hline & 60 & $6.05 \pm 0.05$ & $6.02 \pm 0.01$ & $6.07 \pm 0.02$ & $6.04 \pm 0.02$ & $6.04^{\mathrm{a}} \pm 0.03$ & & & \\
\hline Mean & & $6.04^{\mathrm{a}} \pm 0.02$ & $6.06^{\mathrm{a}} \pm 0.03$ & $6.04^{\mathrm{a}} \pm 0.02$ & $6.05^{\mathrm{a}} \pm 0.03$ & & & & \\
\hline \multirow{3}{*}{$\begin{array}{l}\text { Cooking } \\
\text { Loss }(\%)\end{array}$} & 0 & $27.24 \pm 0.15$ & $26.37 \pm 0.09$ & $28.04 \pm 0.13$ & $26.76 \pm 0.14$ & $27.06^{\mathrm{a}} \pm 0.13$ & & & \\
\hline & 30 & $26.37 \pm 0.47$ & $26.15 \pm 0.06$ & $27.20 \pm 0.16$ & $26.24 \pm 0.04$ & $26.49^{\mathrm{b}} \pm 0.18$ & \multirow{2}{*}{\multicolumn{3}{|c|}{0.59500 .01320 .6951}} \\
\hline & 60 & $27.10 \pm 0.29$ & $27.10 \pm 0.40$ & $27.02 \pm 0.06$ & $26.91 \pm 0.12$ & $27.03^{\mathrm{a}} \pm 0.22$ & & & \\
\hline Mean & & $26.90^{\mathrm{b}} \pm 0.30$ & $26.45^{\mathrm{c}} \pm 0.18$ & $27.42^{\mathrm{a}} \pm 0.12$ & $26.63^{\mathrm{bc}} \pm 0.12$ & & & & \\
\hline
\end{tabular}

Column mean value in each row having different superscript varies significantly at values $\mathrm{p}<0.05$. Again, mean values having the same superscript in each row did not differ significantly at $\mathrm{p}>0.05$. $\mathrm{T}_{1}=$ Control group, $\mathrm{T}_{2}=05 \%$ wheat flour group, $\mathrm{T}_{3}=10 \%$ wheat flour group, $\mathrm{T}_{4}=15 \%$ wheat flour group, $\mathrm{DI}=$ Day Intervals, Treat $=$ Treatment, $\mathrm{T} \times \mathrm{DI}=$ Interaction of Treatment and Day Intervals.

\section{Biochemical properties}

The number of FFA increased with storage time (Table 4). The range of overall observed of different days of intervals of FFA was 0.33 to 0.34 . The FFA value was increased with storage period. The range of overall observed of different days of 
intervals of peroxide value was 3.56 to 3.70. Rhee and Myers (2003) examined peroxide values in plain meatloaf made from ground goat meat and reported a similar trend in peroxide value during storage. The number of peroxide values detected in the samples increased. Generally, TBARs levels significantly $(\mathrm{p}<0.05)$ increased with storage time, showing decreasing shelf life. The range of overall observed of different days of intervals of TBARs value was 0.10 to 0.12 .The control sample, without any added antioxidants, showed a higher level of TBA than samples treated with $05 \%, 10 \%, 15 \%$ wheat flour. The TBA value on day 60 was 0.10 for the control samples, $0.11,0.11,0.11$ for those treated with $05 \%, 10 \%$, and $15 \%$ wheat flour. The different superscript was observed from four treatment groups indicates there were significant differences of TBA value among these four treatments. Racanicci et al. (2004) also found increasing thiobarbituric acid reactive substances (TBARS) as storage time increased to 8 days.

Table 4. Effect of wheat flour on biochemicalparameters in chicken meatballs

\begin{tabular}{|c|c|c|c|c|c|c|c|c|c|}
\hline \multirow{2}{*}{ Parameters } & \multirow{2}{*}{ DI } & \multicolumn{4}{|c|}{ Treatments } & \multirow{2}{*}{ Mean } & \multicolumn{3}{|c|}{ Level of significance } \\
\hline & & $\mathrm{T}_{1}$ & $\mathrm{~T}_{2}$ & $\mathrm{~T}_{3}$ & $\mathrm{~T}_{4}$ & & Treat. & DI & $\mathrm{T} \times \mathrm{DI}$ \\
\hline \multirow{3}{*}{ FFA (\%) } & 0 & $0.33 \pm 0.33$ & $0.33 \pm 0.01$ & $0.31 \pm 0.00$ & $0.34 \pm 0.01$ & $0.33^{\mathrm{a}} \pm 0.00$ & \multirow{3}{*}{0.0725} & \multirow{3}{*}{0.2377} & \multirow{3}{*}{0.1632} \\
\hline & 30 & $0.33 \pm 0.00$ & $0.31 \pm 0.00$ & $0.34 \pm 0.02$ & $0.33 \pm 0.01$ & $0.33^{\mathrm{a}} \pm 0.00$ & & & \\
\hline & 60 & $0.34 \pm 0.00$ & $0.32 \pm 0.01$ & $0.34 \pm 0.01$ & $0.35 \pm 0.00$ & $0.34^{\mathrm{a}} \pm 0.00$ & & & \\
\hline Mean & & $0.33^{\mathrm{ab}} \pm 0.00$ & $0.32^{\mathrm{b}} \pm 0.00$ & $0.33^{\mathrm{ab}} \pm 0.01$ & $0.33^{\mathrm{a}} \pm 0.00$ & & & & \\
\hline \multirow{3}{*}{$\begin{array}{l}\text { PV } \\
\text { (meq/kg) }\end{array}$} & 0 & $3.86 \pm 0.10$ & $3.82 \pm 0.14$ & $3.55 \pm 0.05$ & $3.08 \pm 0.08$ & $3.70^{\mathrm{a}} \pm 0.09$ & \multirow{3}{*}{0.0041} & \multirow{3}{*}{0.2436} & \multirow{3}{*}{0.0005} \\
\hline & 30 & $3.70 \pm 0.10$ & $3.66 \pm 0.16$ & $3.08 \pm 0.08$ & $3.82 \pm 0.14$ & $3.57^{\mathrm{a}} \pm 0.12$ & & & \\
\hline & 60 & $3.84 \pm 0.07$ & $3.58 \pm 020$ & $3.67 \pm 0.06$ & $3.69 \pm 0.14$ & $3.56^{\mathrm{a}} \pm 0.12$ & & & \\
\hline Mean & & $3.80^{\mathrm{a}} \pm 0.09$ & $3.69^{\mathrm{ab}} \pm 0.17$ & $3.44^{\mathrm{c}} \pm 0.06$ & $3.53^{\mathrm{bc}} \pm 0.12$ & & & & \\
\hline \multirow{3}{*}{$\begin{array}{l}\text { TBARS } \\
\text { (MA/kg) }\end{array}$} & 0 & $0.09 \pm 0.01$ & $0.10 \pm 0.00$ & $0.11 \pm 0.00$ & $0.11 \pm 0.00$ & $0.10^{\mathrm{a}} \pm 0.00$ & \multirow{3}{*}{0.2629} & \multirow{3}{*}{0.1590} & \multirow{3}{*}{0.2759} \\
\hline & 30 & $0.12 \pm 0.00$ & $0.12 \pm 0.00$ & $0.11 \pm 0.00$ & $0.11 \pm 0.00$ & $0.12^{\mathrm{a}} \pm 0.00$ & & & \\
\hline & 60 & $0.12 \pm 0.00$ & $0.11 \pm 0.00$ & $0.11 \pm 0.00$ & $0.11 \pm 0.00$ & $0.12^{\mathrm{a}} \pm 0.00$ & & & \\
\hline Mean & & $0.10^{\mathrm{a}} \pm 0.00$ & $0.11^{\mathrm{a}} \pm 0.00$ & $0.11^{\mathrm{a}} \pm 0.00$ & $0.11^{\mathrm{a}} \pm 0.00$ & & & & \\
\hline
\end{tabular}

Column mean value having different superscript varies significantly at values $p<0.05$. Again, mean values having the same superscript in each row did not differ significantly at $\mathrm{p}>0.05 . \mathrm{T}_{1}=$ Control group, $\mathrm{T}_{2}=05 \%$ wheat flour group, $\mathrm{T}_{3}=10 \%$ wheat flour group, $\mathrm{T}_{4}=15 \%$ wheat flour group, DI=Day Intervals, Treat $=$ Treatment, $\mathrm{T} \times \mathrm{DI}=$ Interaction of Treatment and Day Intervals.

\section{CONCLUSION}

The highest amount of dry matter content indicates this product is less preferable but the highest amount of $\mathrm{CP}$ content indicates this product is more preferable. Among four treatments most preferable colour, odour, tenderness, juiciness was observed at $15 \%$ wheat flour group. Therefore, it can be concluded that the $15 \%$ of wheat flour could be used for the production of meatball. 


\section{ACKNOWLEDGEMENT}

We would like to express our appreciation to the Bangladesh Agricultural University Research System (BAURES) for funding this research and their encouragement.

\section{REFERENCES}

Ali, F.H., and Zahran, D.A. (2010).Effect of growth enhancers on quality of chicken meat during cold storage. Advance Journal of Food Science and Technology, 2(4), 219-226.

AOAC. (2016). Official methods of analysis. (20th Edition) Association of official analytical chemists. Washington, DC, USA.

Fernández-López, J., Zhi, N., Aleson, L., Pérez, J.A., and Kuri, V. (2005).Antioxidant and antibacterial activities of natural extracts: Application in beef malondialdehyde and hydroperoxides selectively as a measure of lipid peroxidation. Journal of American Oil Chemists Society, 66, 813-817.

Huang, S.C., Shiau, C.Y., Liu, T.E., Chu, C.L., and Hwang, D.F. (2005).Effects of rice bran on sensory and physicochemical properties of emulsified pork meatballs. MeatScience, 70 (4), 613-619.

Huda, N., Shen, Y.H., and Huey, Y.L. (2009). Proximate composition, colour, texture profile of Malaysian chicken balls. Pakistan Journal of Nutrition, 8 (10), 1555-1558.

Naveena, B.M., Sen, A.R., Vaithiyanathan, S., Babji, Y., and Kondaiah, N. (2008). Comparative efficacy of pomegranate juice, pomegranate rind powder extract and BHT as antioxidants in cooked chicken patties. Meat Science, 80, 1304-1308.

Ngapo, T.M., Dransfield, E., Martin, J.F., Magnusson, M., Bredahl, L., and Nute, G.R. (2004). Consumer perceptions: pork and pig production. Insights from France, England, Sweden and Denmark. Meat Science, 66, 125-134.

Purnomo, A., and Rahardiyan, D. (2008). Indonesian traditional meatball: a review article. International Food Research Journal, 15, 101- 108.

Racanicci, A.M.C., Danielsen, B., Menten, J.F.M., Regitano-D'arce, M.A.B., and Skibsted, L.H. (2004). Antioxidant effect of dittany (Origanumdictamnus) in pre-cooked chicken meatballs during chill-storage in comparison to rosemary (Rosmarinusofficinalis). European Food Research Technology, 218, 521-524.

Rhee, K.S., and Myers, C.E. (2003). Sensory properties and lipid oxidation in aerobically refrigerated cooked ground goat meat. Meat Science, 66, 189-194.

Rukumudin, I.H., White, P.J., Bern, C.J., and Bailey, T.B. (1998).A modified method for determining free fatty acids from small soybean sample sizes. Journal of American Oil Chemists Society, 75, 563-568.

Sallam, K.I., Ishioroshi, M., and Samejima, K. (2004).Antioxidants and antimicrobial effects of garlic in chicken sausage. Food Science and Technology, 37(8), 849-855.

Sampaio, G.R., Castellucci, C.M.N., Elisabeth, M.M., Silva, P.E., and Torres, E.A.F.S. (2004). Effect of fat replacers on the nutritive value and acceptability of beef frankfurters. Journal of Food Composition and Analysis, 17, 469-474. 
Schemedes, A., and Holmer, G.(1989).A new Thiobarbituric acid (TBA) method for determining free malondialdehyde and hydroperoxides selectively as a measure of lipid peroxidation. Journal of the American Oil Chemists Society, 66 (6), 813-817.

Serdaroglu, M., Yildiz-Turp, G., and Abrodimov, K.(2005). Quality of low-fat meatballs containing legume flours as extenders. Meat Science, 70, 99-105.

Zhang, H., Boahua, K., Youn, L.X., Ling and Sun, X. (2009). Antimicrobial activities of spices extracts against pathogenic and spoilage bacteria in modified atmosphere packaged fresh pork and vacuum packaged ham slices stored at $4 \mathrm{O}^{0} \mathrm{C}$. Meat Science, 81(4), 686-692. 